\title{
High duty cycle, highly efficient fiber coupled 940-nm pump module for high-energy solid-state lasers
}

\author{
René Platz, Bernd Eppich, Juliane Rieprich, Wolfgang Pittroff, Götz Erbert, and Paul Crump \\ Ferdinand-Braun-Institut, Leibniz-Institut für Höchstfrequenztechnik, Gustav-Kirchhoff-Straße 4, 12489 Berlin, Germany \\ (Received 30 November 2015; revised 8 January 2016; accepted 26 January 2016)
}

\begin{abstract}
Tailored diode laser single emitters with long $(6 \mathrm{~mm})$ resonators and wide $(1.2 \mathrm{~mm})$ emission apertures that operate with $940 \mathrm{~nm}$ emission wavelength were assembled in novel edge-cooled vertically stacked arrays, and used to construct a compact and highly efficient fiber coupled pump source for Yb:YAG pulsed high-energy class solid-state lasers. The novel configuration is shown to allow repetition rates of $200 \mathrm{~Hz}$ at $1 \mathrm{~ms}$ pulse duration, at an output power of $130 \mathrm{~W}$ per single emitter. The emission of two stacked arrays was then optically combined to realize pump modules that deliver $6 \mathrm{~kW}$ peak power (pulse energy $6 \mathrm{~J}$ ) from a $1.9 \mathrm{~mm}$ core diameter fiber, with wall plug efficiency of $50 \%$. This represents a significant improvement in terms of duty cycle and electro-optical efficiency over conventional sources. The pump module has been successfully tested at the Max Born Institute, Berlin during trials for pumping of disk lasers.
\end{abstract}

Keywords: diode laser; diode pumped solid-state laser; fiber coupling; high-power; QCW diode laser stack

\section{Introduction}

High-energy class diode pumped solid-state lasers (HECDPSSLs) with pulse energies $>1 \mathrm{~J}$ (ns- to fs-pulses) and average powers of several hundred watts are increasingly required in industry, science and basic research ${ }^{[1,2]}$. Fields of applications include laser shock peening, material analytics, attosecond spectroscopy or particle acceleration. However, the achievable performance of such a system is limited by the characteristics of the diode laser pump sources. The diode lasers are operated in quasi-continuous wave (QCW) mode, with pulse widths matched to the upper state lifetime of the crystal in use. Commercially available multi-kW QCW diode pump sources are based on low-cost vertical stacked diode laser bars with inefficient rear side cooling. Typically, they operate at maximum pulse width $\tau=1 \mathrm{~ms}$ and maximum repetition rate $f=10 \mathrm{~Hz}$, corresponding to a duty cycle $d c=1 \%$ (see for example Refs. [2,3]). However, repetition rates of several hundred $\mathrm{Hz}$ are required to boost average power from the DPSSL and to increase the shot frequency ${ }^{[4]}$. Although very high-power continuous wave diode laser pump sources are commercially available and can be used for power scaling, they are typically not preferred as they can lead to overheating of the YAG crystal ${ }^{[5]}$. Since the lifetime of the diode laser stacks is a complex function

Correspondence to: R. Platz. Ferdinand-Braun-Institut, Leibniz-Institut für Höchstfrequenztechnik, Gustav-Kirchhoff-Straße 4, 12489 Berlin, Germany. Email: rene.platz@fbh-berlin.de of QCW pulse conditions and stack construction ${ }^{[6]}$, careful mechanical design is needed to ensure reliability under the required operational conditions. In all cases, high-power conversion efficiency and a compact, low-cost mechanical design are important.

Based on a novel approach using tailored single emitters with wide lateral aperture ${ }^{[7-10]}$, we present a QCW fiber coupled pump module with operating wavelength $\lambda=940 \mathrm{~nm}$ and $\sim 6 \mathrm{~kW}$ output power allowing efficient operation at duty cycles $10 \%-20 \%$ ( $\tau=1 \mathrm{~ms}$ ). The module has been designed for use with a high average power Yb:YAG thin disk laser system at the Max Born Institute $(\mathrm{MBI})^{[4]}$, that produces picosecond pulses with multi-joule energies at repetition rates up to $200 \mathrm{~Hz}$. Details of the design concept were previously presented in Ref. [10], which focused on the performance of the wide aperture single emitters, that were packaged in a 'sandwich' configuration into a doublesided passive heat sink, and cooled from the device edges. In the present paper, we first briefly summarize the diode laser and stack performance, then present results on the design used to optically combine the emission from two stacks into a $1.9 \mathrm{~mm}$ core fiber, reviewing the performance achieved. Finally we give details of full characterization of complete integrated pump modules, confirming that the beam delivered from the fiber is suitable for use in high repetition rate pumping of disk lasers. 


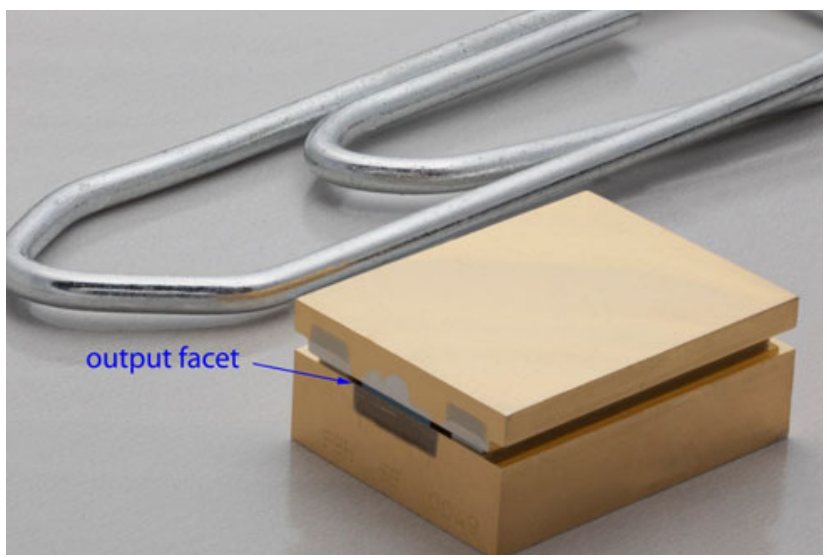

Figure 1. Single stack element.

\section{Chip design and mounting}

\subsection{Chip design}

As described in Ref. [10], the laser chip was optimized for QCW operation at $\lambda=940 \mathrm{~nm}$ and both high output power and high efficiency. The vertical design incorporates an extreme double-asymmetric super large optical cavity (EDASLOC) epitaxial structure consisting of an InGaAs double quantum-well (DQW) embedded in a $3.6 \mu \mathrm{m}$ thick AlGaAs waveguide. The contact layer was structured into $5 \mu \mathrm{m}$ wide stripes with $10 \mu \mathrm{m}$ spacing (aperture width $W=1200 \mu \mathrm{m}$ ), which were defined by shallow proton implantation. The facets of the laser chip, with total width $2 \mathrm{~mm}$ and cavity length $L=6 \mathrm{~mm}$, were passivated and coated with a reflectivity of $R_{f}=0.9 \%$ at the front side and $R_{r}=96 \%$ at the rear side. The final laser chip was then mounted in 'sandwich' configuration between a $2 \mathrm{~mm}$ and $1 \mathrm{~mm}$ thick expansion matched $\mathrm{CuW}$ heat sink using AuSn solder (Figure 1), to produce a single stack element. These elements are characterized in QCW mode using a clamping test fixture, at a temperature of $25^{\circ} \mathrm{C}$. Each element operates at $P_{\text {out }}=130 \mathrm{~W}$ and $60 \%$ conversion efficiency $(\tau=1 \mathrm{~ms}$, $f=100-200 \mathrm{~Hz}$ ). This corresponds to a power density of $>1 \mathrm{~kW}$ per $\mathrm{cm}$ aperture, which is more than double that of conventional commercial QCW bars (300-400 W/cm aperture). The measured vertical and lateral divergence angles are $\theta_{V} \sim 43^{\circ}$ and $\theta_{L} \sim 12^{\circ}$ (95\% power included), respectively at the nominal output power of $130 \mathrm{~W}$. The lateral beam parameter product per stack element at the operation point is $\mathrm{BPP}_{L}=0.25 \times W \times \theta_{L}=63 \mathrm{~mm} \mathrm{mrad}$, accordingly the beam propagation ratio is $M^{2} \sim 220$.

\subsection{Stack design}

The sandwich mounts described in Section 1 were subsequently used as building blocks (stack elements) for a diode laser stack consisting of 28 emitters with vertical

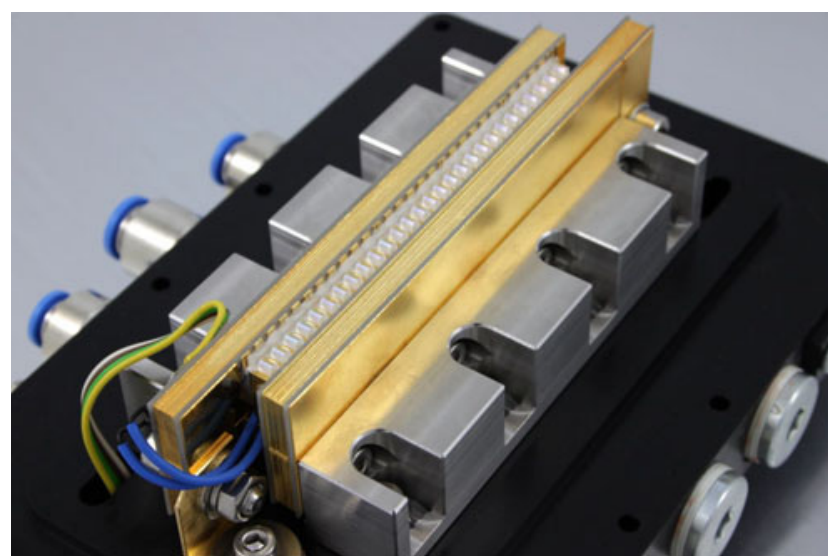

Figure 2. $3.6 \mathrm{~kW}$ stack subassembly with fast-axis collimation.

Table 1. Typical stack data (measured).

\begin{tabular}{lc}
\hline Number of emitters & 28 \\
Operating current & $140 \mathrm{~A}$ \\
Pulse width & $1 \mathrm{~ms}$ \\
Repetition rate & $100-200 \mathrm{~Hz}$ \\
Peak power & $3.6 \mathrm{~kW}$ \\
Electro-optical efficiency & $60 \%$ \\
Vertical BPP & $<90 \mathrm{~mm} \mathrm{mrad}\left(M^{2}<300\right)$ \\
Lateral BPP & $<90 \mathrm{~mm} \mathrm{mrad}\left(M^{2}<300\right)$ \\
\hline
\end{tabular}

spacing of $3.16 \mathrm{~mm}$. Furthermore, for lateral heat dissipation, water-cooled DCB coolers were mounted to both sides of the stack using soft solder, which takes advantage of the long resonator length to cool the single emitters efficiently, leading to both low electrical and low thermal resistance $^{[7,10]}$. The thermal resistance of a single stack element is $1.7 \mathrm{~K} / \mathrm{W}$, determined by tracking the change in centroid wavelength with varying bias in QCW mode ( $\tau=1 \mathrm{~ms}, f=100 \mathrm{~Hz}$ ). The use of expansion matched coolers in sandwich configuration and distributed cooling along the length of the single emitter minimize temperature gradients and mechanical stress, for enhanced performance, reliability and efficiency. Figure 2 shows the complete stack subassembly with fast-axis collimation (FAC). A summary of key performance characteristics data is given in Table 1 .

The lateral BPP of the stack turned out to be significantly larger than the BPP of the single stack elements. The reason for this behavior is not clear and under current investigation. One possible reason is optical feedback (most likely from the FAC lenses) into the laser resonators causing the excitation of higher-order optical modes with higher divergence angles.

\section{Fiber coupling}

We developed an optical design based on simple geometrical coupling $^{[7,10]}$, allowing for low-cost manufacturing and featuring high robustness. As noted in Section 2.2, each single 


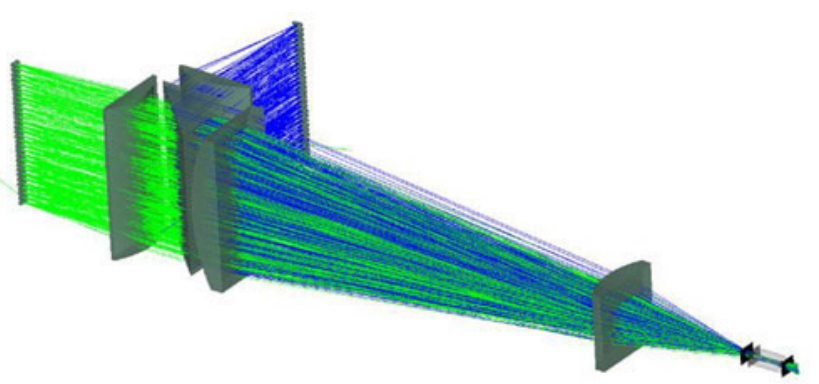

Figure 3. Optical simulation of the $6 \mathrm{~kW}$ fiber coupling system.

stack element was vertically collimated using individual FAC lenses (with effective focal length $1.2 \mathrm{~mm}$ ). Lateral focusing was achieved using a common acylindrical lens. The beams of two stacks are geometrically combined using a stack of thin right-angle prism plates, as illustrated in the optical simulation shown in Figure 3. The combined beam is subsequently coupled into a custom high-power optical fiber ${ }^{[11]}$ with core diameter $D=1.9 \mathrm{~mm}$ and numerical aperture $N A=0.22$, by using one vertical acylindrical and one lateral cylindrical lens. All lenses as well as prism elements were fabricated with anti-reflective (AR) coatings, to minimize back-reflections. Geometrical coupling efficiency was estimated by forming an image of the beam into the expected fiber input plane into a high spatial resolution camera, as shown in Figure 4. An integration of the imaged beam profile at the fiber entry point implies a measured geometrical coupling efficiency of $>98 \%$, which is in good agreement with the measured total optical-to-optical coupling efficiency of $\sim 88 \%$, taking into account $4 \%$ Fresnel losses at each fiber end, since the fibers were not AR coated, and $3 \%$ internal losses in the fiber, which is a characteristic of the custom fiber used (manufacturers data).

Following standard practice, in order to evaluate the achieved beam quality, we determined the beam propagation factor $M^{2}$ of the geometrically combined stacks by a caustic measurement. This has been done by measuring the beam diameter at different longitudinal positions around the beam waist, with results shown in Figure 5 for the full complete system, containing all elements except for the fiber. The $M^{2}$ values in lateral and vertical direction were obtained by a fit to the measurement data. The values achieved are $M^{2} \sim 330$ (lateral) and $M^{2} \sim 360$ (vertical), which is close to the measured $M^{2}$ of the single stacks before beam combination. The results indicate that the tolerances of the fast-axis collimation, regarding beam direction and beam divergence, as well as the vertical stack adjustment are sufficient for the collimated beams to pass the prism stack without significant degradation of beam quality. In addition, no fine alignment of the position and orientation of the large coupling lenses was necessary, showing the relaxed tolerances of the complete beam forming system. The measured $M^{2}$ is only half of the $M^{2}$ of the fiber $\left(M^{2} \sim 700\right)$

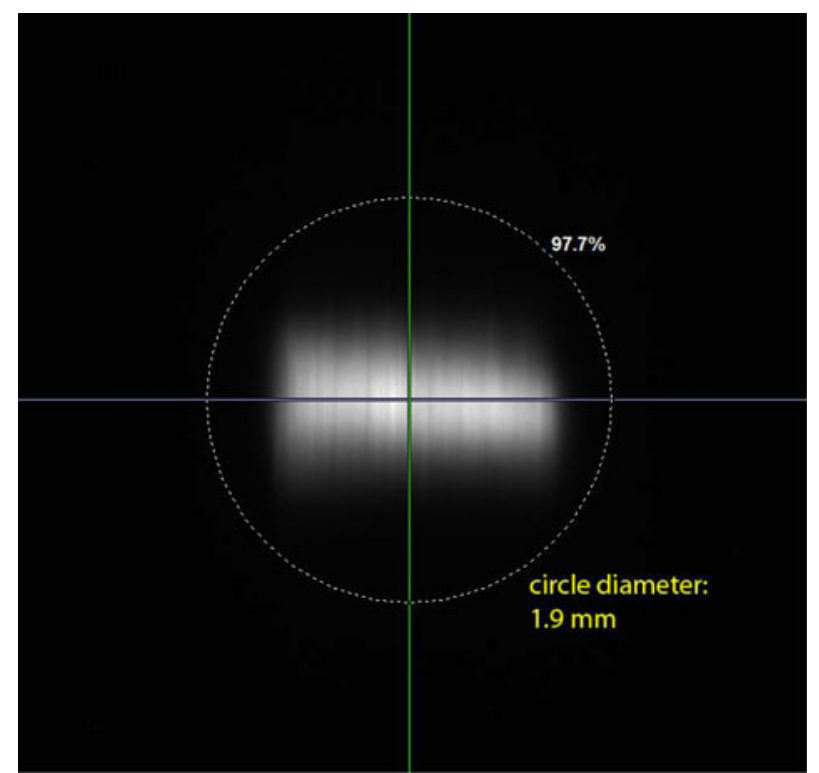

Figure 4. Measured beam profile near the focus plane, which is identical with the coupling plane. The plotted circle corresponds to the fiber core diameter.

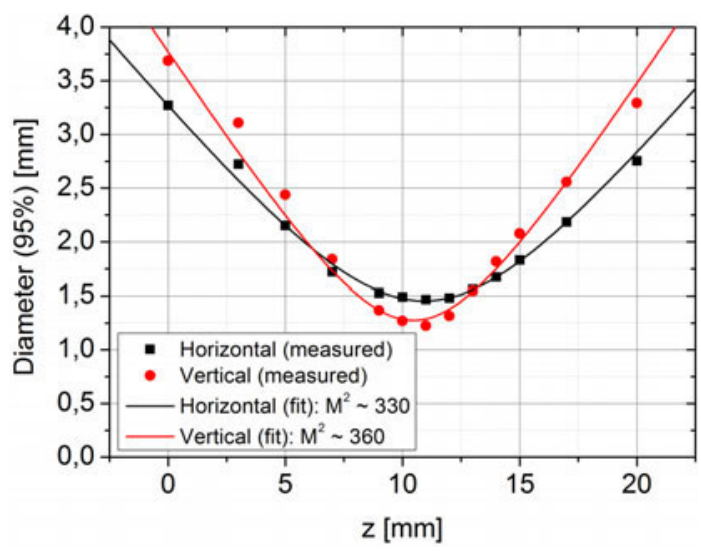

Figure 5. Measured caustic of the focused laser beam.

allowing efficient fiber coupling. It should also be noted that the system should also be compatible (when appropriate beam forming is used) with a standard $1.5 \mathrm{~mm}$ core diameter fiber $\left(M^{2} \sim 550\right)$, although this was not attempted, as it is not necessary for the current application.

\section{Output characteristics}

Figure 6 shows the completely assembled $6 \mathrm{~kW}$ pump module. The two stacks have separate water-cooling and are separately driven each with a high-current pulse driver with maximum duty cycle $d c=10 \%$. The fiber connector and the fiber mounting flange are also water-cooled. The power characteristics are shown in Figure 7. The measurement was 


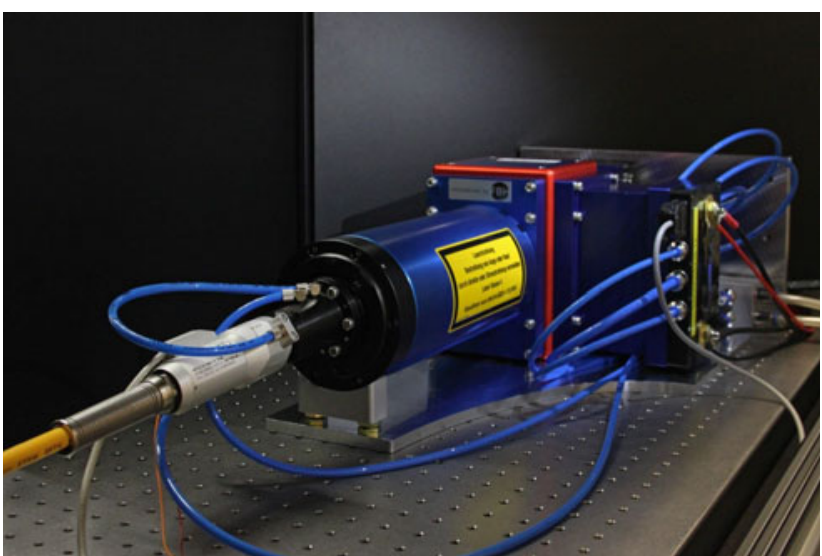

Figure 6. $6 \mathrm{~kW}$ pump module with $1.9 \mathrm{~mm}$ high-power fiber. Dimensions without fiber: $68 \mathrm{~cm} \times 32 \mathrm{~cm} \times 20 \mathrm{~cm}$.

conducted with $\tau=1 \mathrm{~ms}, f=100 \mathrm{~Hz}(d c=10 \%)$ and cooling water temperature $T=17^{\circ} \mathrm{C}$. We measured the average optical power $\left\langle P_{\text {out }}\right\rangle$ using a Gentec high-power thermodetector and calculated peak power $P_{\text {out }}=\left\langle P_{\text {out }}\right\rangle / d c$. At operating current $I=140 \mathrm{~A}$, the pump module reaches $P_{\text {out }}=6 \mathrm{~kW}$, corresponding to a pulse energy $E_{\mathrm{p}}=6 \mathrm{~J}$, and electro-optical efficiency $\eta=50 \%$. The performance was subsequently remeasured on a separate test station, in dependence on duty cycle, as shown in Figure 8(a), with higher duty cycle not significantly lowering $P_{\text {out }}$. (Small differences in total power between Figures 7 and 8(a) are due to an offset in calibration of the two test stations.) The temperature measured at the top of the stack (with in-line Pt100 sensor $)$ did not exceed $30^{\circ} \mathrm{C}(I=140 \mathrm{~A}, d c=20 \%)$. The optical spectrum is shown in Figure $8(\mathrm{~b})$. It can be seen that the center wavelength shifts from $\lambda=937.5 \mathrm{~nm}$ at $d c=10 \%$ to $\lambda=939.5 \mathrm{~nm}$ at $d c=20 \%$. This is consistent with a temperature rise $\Delta T \approx \Delta \lambda /(0.3 \mathrm{~nm} / \mathrm{K})=10 \mathrm{~K}$ in the average temperature of the diode lasers. Spectral width with $95 \%$ power content is $7 \mathrm{~nm}$. This is wider than the typical $5 \mathrm{~nm}$ for a single stack element, potentially due to chip to chip variation, but consistent with the requirements for pumping the broad $\mathrm{Yb}$ :YAG absorption band at $940 \mathrm{~nm}$. All modules showed stable operation over more than $500 \mathrm{~h}$ of operation. Life tests with single stack elements over $1000 \mathrm{~h}$ of operation were also performed without failure ${ }^{[10]}$, as were initialization tests of the pump module units over $12 \mathrm{~h}$ of operation $(50-100 \mathrm{~Hz}, 130 \mathrm{~A})$. In continuous wave mode, diode lasers assembled using comparable epitaxial designs and process technology were shown to operate for many $1000 \mathrm{~h}$ in continuous wave mode at much higher optical intensities ( $7 \mathrm{~W}$ per $30 \mu \mathrm{m}$ output aperture ${ }^{[12]}$, more than double optical intensity to that used here). Therefore, the chip technology is anticipated to enable a lifetime of many $1000 \mathrm{~h}$ in QCW mode, but special aging studies are necessary to confirm this for the complete systems.

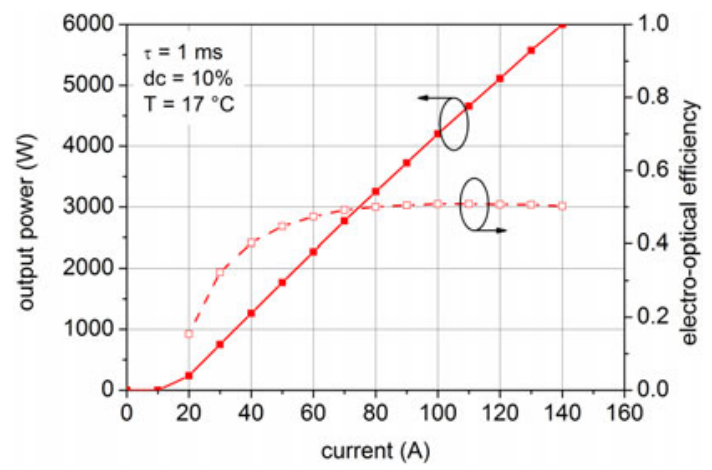

Figure 7. Power characteristics of the pump module (out of fiber).
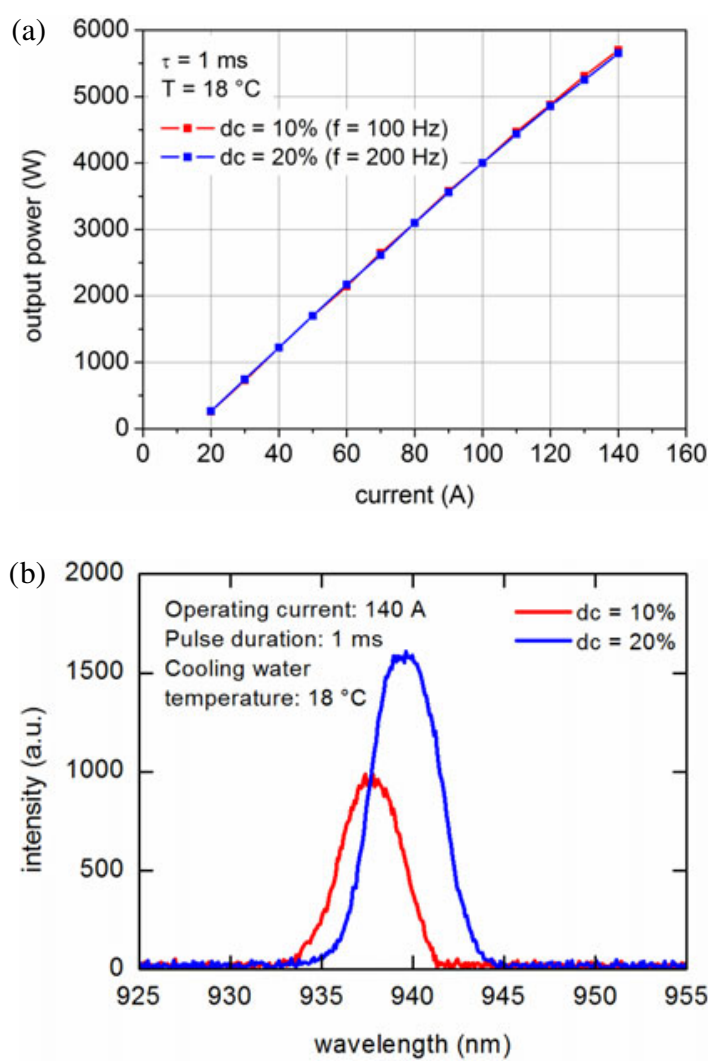

Figure 8. Peak output power (a) and spectral distribution (b) of the pump module depending on duty cycle.

\section{Conclusions}

We have presented a robust and compact $6 \mathrm{~kW}$, high duty cycle fiber coupled diode laser module with $50 \%$ power conversion efficiency for pumping Yb:YAG based HECDPSSL systems. The base component of the pump module is a side-cooled diode laser stack comprising 28 single emitters with $1.2 \mathrm{~mm}$ lateral aperture. A simple optical design with minimal number of optical components and relaxed tolerances enables efficient fiber coupling and lowcost manufacturing. 
The measured beam propagation parameter of the complete modules, the single stacks and particularly the individual stack elements indicates the possibility of coupling into significant smaller fiber cores.

\section{Acknowledgments}

The authors thank the Max Born Institute for measurements of complete pump modules as a function of duty cycle as well as $\mathrm{C} 2 \mathrm{GO}$ inprocess solutions $\mathrm{GmbH}$ for the mechanical design and manufacture of the housing for the pump module.

This work was funded in part via the European Regional Development Fund (ERDF) under contract number $200720132 / 42$.

\section{References}

1. M. Divoky, M. Smrz, M. Chyla, P. Sikocinski, P. Severova, O. Novak, J. Huynh, S. S. Nagisetty, T. Miura, J. Pilar, O. Slezak, M. Sawicka, V. Jambunathan, J. Vanda, A. Endo, A. Lucianetti, D. Rostohar, P. D. Mason, P. J. Phillips, K. Ertel, S. Banerjee, C. Hernandez-Gomez, J. L. Collier, and T. Mocek, High Power Laser Sci. Eng. 2, e14 (2014).

2. C. Wessling, O. Rübenach, S. Hambücker, V. Sinhoff, S. Banerjeea, K. Ertel, and P. Mason, Proc. SPIE 8602, 86020I (2013).
3. H. Kissel, W. Fassbender, J. Lotz, K. Alegria, T. Koenning, D. Stapleton, S. Patterson, and J. Biesenbach, Proc. SPIE 8605, 86050V (2013).

4. http://www.mbi-berlin.de/de/research/projects/1.2/topics/1_p ower_disk_laser/ (2015).

5. S. Klingebiel, M. Schultze, C. Y. Teisset, R. Bessing, M. Häfner, S. Prinz, M. Gorjan, D. Sutter, K. Michel, H. G. Barros, Z. Major, F. Krausz, and T. Metzger, in CLEO: Science and Innovations 2015 OSA Technical Digest (Online), (Optical Society of America, San Jose, CA, USA, 2015), paper STu4O2.

6. Y. Berk, Y. Karni, G. Klumel, M. Levy, Y. Openhaim, S. Risemberg, M. Rech, H. Becht, B. Frei, and F. Monti di Sopra, Proc. SPIE 7198, 71980C (2009).

7. W. Pittroff, G. Erbert, B. Eppich, C. Fiebig, K. Vogel, and G. Tränkle, IEEE Trans. Compon. Packag. Technol. 33, 1, 206 (2010).

8. R. Platz, G. Erbert, W. Pittroff, M. Malchus, K. Vogel, and G. Tränkle, High Power Laser Sci. Eng. 1, 60 (2013).

9. R. Platz, B. Eppich, P. Crump, W. Pittroff, S. Knigge, A. Maaßdorf, and G. Erbert, IEEE Photonics Technol. Lett. 26, 6, 625 (2014).

10. W. Pittroff, B. Eppich, G. Erbert, R. Platz, D. Tyralla, and G. Traenkle, Proc. SPIE 8965, 896515 (2014).

11. Fiber fabricated by Frank Optic Products GmbH, www.frankoptic-products.com (2015).

12. St. Knigge, P. Crump, H. Wenzel, G. Erbert, and G. Tränkle, in IEEE Photonics Conference (IEEE, Bellevue, WA, USA, 2013), pp. 466-467. 